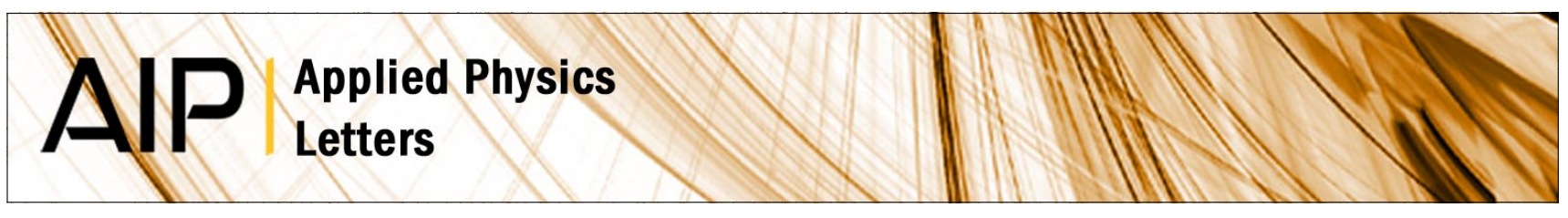

\title{
Zero-point oscillations in the vicinity of atoms
}

Alexander S. Shumovsky

Citation: Appl. Phys. Lett. 79, 464 (2001); doi: 10.1063/1.1384006

View online: http://dx.doi.org/10.1063/1.1384006

View Table of Contents: http://apl.aip.org/resource/1/APPLAB/v79/i4

Published by the American Institute of Physics.

Additional information on Appl. Phys. Lett.

Journal Homepage: http://apl.aip.org/

Journal Information: http://apl.aip.org/about/about_the_journal

Top downloads: http://apl.aip.org/features/most_downloaded

Information for Authors: http://apl.aip.org/authors

\section{ADVERTISEMENT}

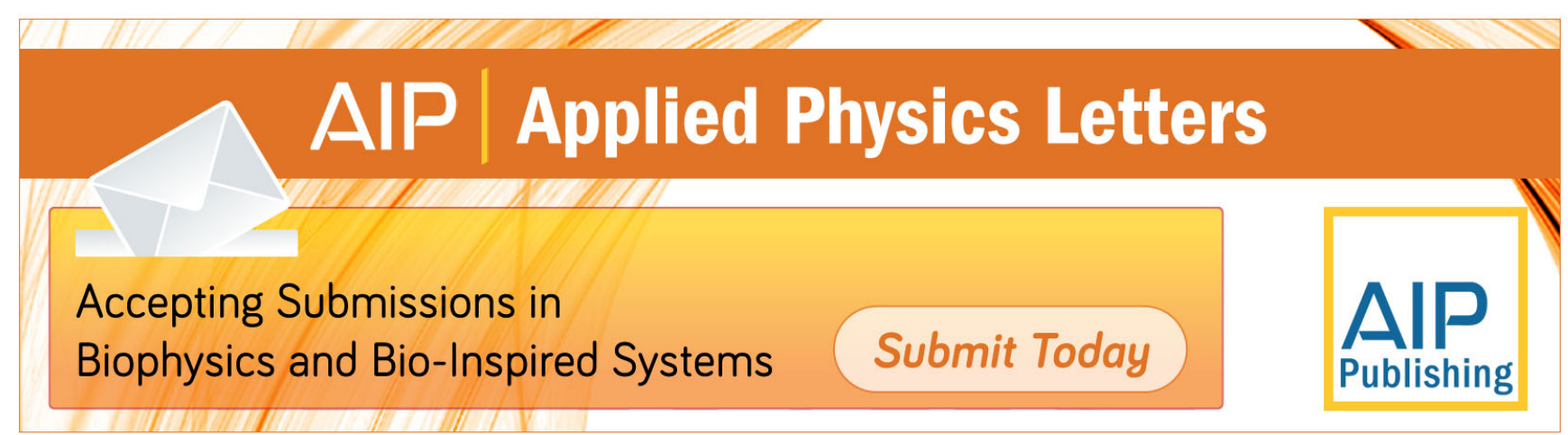




\title{
Zero-point oscillations in the vicinity of atoms
}

\author{
Alexander S. Shumovskya) \\ Physics Department, Bilkent University, Bilkent, Ankara, 06533, Turkey
}

(Received 9 October 2000; accepted for publication 16 May 2001)

It is shown that the vacuum fluctuations of an electromagnetic field are concentrated near atoms.

This effect worsens the quantum limit of precision of the measurements in atomic systems.

(c) 2001 American Institute of Physics. [DOI: 10.1063/1.1384006]

It is well known that the quantum nature of electromagnetic radiation manifests itself in the zero-point oscillations (ZPO) of the field strengths. In particular, ZPO are responsible for a number of important effects such as the spontaneous emission, Lamb shift, Casimir-Polder force, quantum beats, etc. (e.g., see Refs. 1-3). As a source of quantum noise, ZPO define the limit of precision of quantum measurements. ${ }^{2-4}$ In the usual treatment, ZPO are calculated as though the field is represented by the plane waves of photons in empty space. ${ }^{1-4}$ This simplified picture overlooks the fact that the atomic transitions emit the multipole radiation ${ }^{5}$ represented by the quantized spherical waves. ${ }^{6}$

The purpose of this letter is to trace the difference between the ZPO of plane waves in empty space and those of spherical waves of photons in presence of atoms. Such an investigation, although simple in itself, seems to be important for the experiments with single-atom masers, ${ }^{7}$ trapped Ridberg atoms, ${ }^{8}$ and for estimation of the Casimir-Polder forces between atoms. ${ }^{1}$ This issue has attracted a great deal of interest in connection with the general problems of quantum physics as well as with applications in the field of optical communication and information technologies.

The qualitative difference between the ZPO of plane and multipole waves immediately follows from the comparison of energies of free fields, described by well known formulas $^{2-4}$

$$
H_{\text {(plane) }}=\sum_{k, \sigma} \hbar \omega_{k}\left(a_{k \sigma}^{+} a_{k \sigma}+1 / 2\right)
$$

and $^{5}$

$$
H_{\text {(multi) }}=\sum_{k} \sum_{\lambda, j, m} \hbar \omega_{k}\left(a_{k \lambda j m}^{+} a_{k \lambda j m}+1 / 2\right) .
$$

Here $\sigma=1 / 2$ is the index of polarization of plane waves, $\lambda$ labels the type of multipole radiation (either electric or magnetic), $j=1,2, \ldots$ is the angular momentum of photons, $m$ $=-j, \ldots, j$ is the projection of the angular momentum, and $a_{(\cdot)}$ denotes the corresponding photon annihilation operator. Then, the energy of the ZPO (the energy of the vacuum state) is

$$
H_{(\text {plane) }}^{(\mathrm{vac})}=\sum_{k, \sigma} \hbar \omega_{k} / 2=\sum_{k} \hbar \omega_{k}
$$

${ }^{a)}$ Electronic mail: shumo@fen.bilkent.edu.tr

$$
H_{(\mathrm{multi})}^{(\mathrm{vac})}=\sum_{k} \sum_{\lambda, j, m} \hbar \omega_{k} / 2=\sum_{k}\left[\sum_{j=1}^{\infty}(2 j+1) \hbar \omega_{k}\right] .
$$

At first sight, Eqs. (3) and (4) are equivalent because both give the infinite energy of the vacuum state. In fact, this infinity is inessential because of the following reason. The point is that the contribution of the ZPO can be recognized only through a measurement which implies an averaging of physical quantities over a finite "volume of detection" and exposition time of detector. ${ }^{9}$ In other words, any real measurement involves a filtration, leading to a separation of finite transmission frequency band (TFB).

It is seen from Eqs. (3) and (4), that even if we assume that the filtration process separates the dipole photons only, the right-hand side in Eq. (4) exceeds that in Eq. (3) in three times (at the same TFB). If we further restrict consideration by the electric dipole photons $(\lambda=E$ and $j=1)$, the ratio between the Eqs. (3) and (4) is

$$
\left[H_{(\text {multi) }}^{\mathrm{vac}} / H_{(\text {plane })}^{\mathrm{vac}}\right]_{(\lambda=E, j=1)}=3 / 2 \text {. }
$$

Thus, the measuring level of the ZPO of multipole radiation exceeds that of the plane waves of photons. From the physical point of view, this result is caused by the fact that the multipole field is specified by more quantum degrees of freedom than the plane waves of photons and each degree of freedom contributes into the vacuum fluctuations.

We now stress that the expressions (3), (4), and (5) correspond to the vacuum state energy in the whole volume of quantization. A much more interesting and important result can be obtained from the consideration of spatial properties of the field.

The plane waves of photons in a finite volume $V$ are specified by the following positive-frequency part of the vector potential ${ }^{2,3}$

$$
\mathbf{A}_{\text {(plane) }}^{(+)}(\mathbf{r}, t)=\sum_{k, \sigma} \gamma_{k} \mathbf{e}_{k \sigma} e^{i \mathbf{k} \cdot \mathbf{r}} e^{-i \omega_{k} t} a_{k \sigma},
$$

where $\gamma_{k}=\sqrt{2 \pi \hbar \omega_{k} / k^{2} V}$ is the normalization factor and $\mathbf{e}_{k \sigma}$ denotes the unit vector of polarization. In turn, for the multipole radiation, we have

$$
\mathbf{A}_{(\text {multi) }}^{(+)}(\mathbf{r}, t)=\sum_{k, \lambda, j, m} \gamma_{k} \sum_{\mu=-1}^{1} \boldsymbol{\epsilon}_{\mu} V_{k \lambda j m \mu}(\mathbf{r}) e^{-i \omega_{k} t} a_{k \lambda j m} .
$$

Here, $\boldsymbol{\epsilon}_{\mu}$ denotes the base vectors of the so-called helicity basis, ${ }^{10}$ centered at the local source (atom). In the case of radiation in a cavity, the mode functions $V(\mathbf{r})$ are expressed 


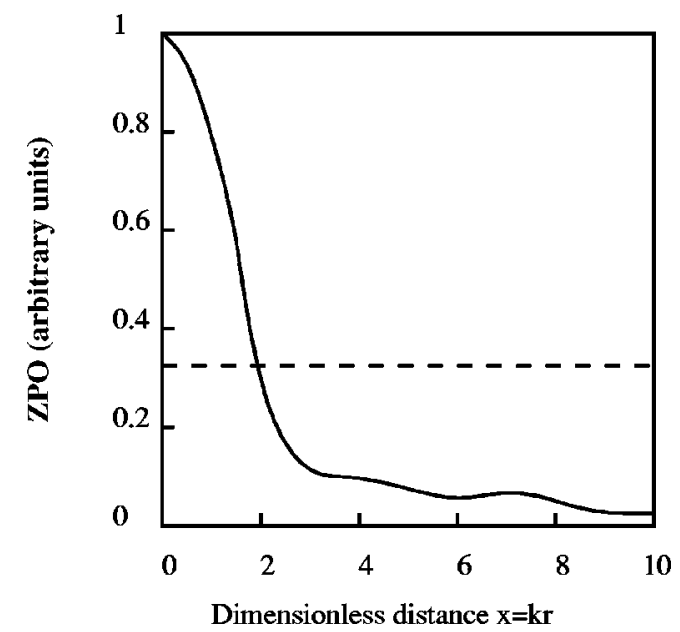

FIG. 1. The ZPO of plane waves (dotted line) and multipole waves vs dimensionless distance $k r$ are shown.

in terms of linear combinations of spherical Bessel functions $j_{l}(k r) \quad(l=j, j \pm 1), \quad$ Clebsch-Gordon coefficients, and spherical harmonics. ${ }^{6,11}$ Independent of the representation, the density of the ZPO is specified by the following commutator $^{4}$

$$
\left.W_{(\cdot)}^{(\mathrm{vac})}=\frac{k^{2}}{8 \pi}\left[\mathbf{A}^{(+}\right)(\mathbf{r}, t), \mathbf{A}^{(-)}(\mathbf{r}, t)\right],
$$

where $\mathbf{A}^{(-)}=\left(\mathbf{A}^{(+)}\right)^{+}$. In the case of plane waves, it leads to

$$
W_{(\text {plane })}^{(\text {vac) }}=\frac{k^{2}}{8 \pi} \sum_{k, \sigma} \gamma_{k}^{2} .
$$

It is seen that, in spite of the position dependence of the mode function, the energy density of the ZPO in Eq. (9) is spatially homogeneous. Unlike the result in Eq. (9), in the case of multipole field, Eq. (8) takes the form

$$
W_{(\text {multi) }}^{(\mathrm{vac})}(\mathbf{r})=\sum_{k} \gamma_{k}^{2} \sum_{\lambda, j, m, \mu}\left|V_{k \lambda j m \mu}(\mathbf{r})\right|^{2}
$$

The spatial inhomogeneity of multipole ZPO comes from the position dependence of the mode function $V(\mathbf{r})$. Taking into account the properties of spherical harmonics and ClebschGordon coefficients, it is a straightforward matter to show that $|V(\mathbf{r})|^{2}$ in Eq. (10) is independent of the angular variables. The right-hand side in Eq. (10) tends to Eq. (9) at far distances $k r \gg 1$. Hence, the ZPO are distributed symmetrically with respect to the local source (atom) and are concentrated in some neighborhood of the source where they can strongly exceed the level of Eq. (9) predicted by the model of plane waves of photons.

The result can be illustrated by Fig. 1, showing the contribution of the dipole terms with $j=1$ into Eq. (10) versus dimensionless distance $k r$ from the source in the case of a monochromatic field. The dotted line shows the level of the ZPO of Eq. (9). It is seen that the ZPO of Eq. (10) are concentrated at least in the region of the order of $r \leqslant r_{0}$ $\sim 2 / k=\lambda / \pi$ around the atom, where $\lambda$ denotes the wavelength. Thus, the effect of condensation of the vacuum noise near atoms can be observed in the near and intermediate zones. Therefore, the effect seems to be important for the near-field optics. We note that a qualitatively similar result can be obtained for the outgoing and incoming spherical waves described by the spherical Hankel functions. In this case, to avoid the divergence at $k r \rightarrow 0$, we have to assume that the atom occupies a finite volume.

To stress the importance of the obtained result, we now note that, in a number of modern experiments on engineered entanglement in the system of trapped Ridberg atoms, the interatomic distances are of the order of $r_{0}$ or even less. ${ }^{8}$ Thus, this effect is important for an adequate estimation of the quantum fluctuations of radiation in such systems. Since, in this case, we have more than one atom, consider as an illustrative example the system of two identical atoms separated by distance $d$. Assume that one of the atoms (source) is initially in an excited state, while the other (detector) is in the ground state. The emission of a photon by the source atom and successive absorption by the detecting atom can be interpreted as a Hertz-type measurement. This experiment should be described in terms of the spherical waves of photons as a superposition of outgoing and incoming waves focused on the source and detector, respectively. Such a superposition should obey the boundary conditions for the radiation field. It should be stressed that this quantum picture is insensitive to a "real path" of the photon, while it obeys the causality principle. ${ }^{10}$ It then follows from the aforementioned results that both atoms "condense" the vacuum fluctuations around. If $d \gg r_{0}$, the detection process is influenced mainly by the vacuum noise due to the detecting atom. In the opposite case of short interatomic distances $d \leqslant r_{0}$, there is an overlap of the ZPO concentrated near the source and detector, which worsens the quantum limit of precision of the measurement.

This effect can be important for the polarization entanglement investigation in the systems of trapped Ridberg atoms as well. The point is that the multipole radiation has, at short distances, a linearly polarized longitudinal (radial) component in addition to the circular polarized transversal components (e.g., see Refs. 12 and 13). Therefore, the polarization is described by the $(3 \times 3)$ Hermitian polarization matrix instead of a conventional $(2 \times 2)$ polarization matrix of plane waves. In the quantum case, the elements of the corresponding operator matrix of a monochromatic electrictype $j$-pole field have the form ${ }^{13}$

$$
P_{\mu \mu^{\prime}}(\mathbf{r})=k^{2} A_{\mu}^{(-)}(\mathbf{r}) A_{\mu^{\prime}}^{(+)}(\mathbf{r}),
$$

where $A_{\mu}^{( \pm)}=\boldsymbol{\epsilon}_{\mu}^{*} \times \mathbf{A}^{( \pm)}$. The corresponding ZPO are then described by the following commutators

$$
k^{2}\left[A_{\mu^{\prime}}^{(+)}(\mathbf{r}), A_{\mu}^{(-)}(\mathbf{r})\right],
$$

similar to Eq. (8). Following this consideration, it is a straightforward matter to arrive at the conclusion that the vacuum fluctuations of polarization are also condensed near the atoms where they can strongly influence the precision of the polarization measurements. The "shot noise limit" determined by these strong fluctuations should be taken into account in the design of experiments on polarization entanglement in atomic systems.

The aforementioned effect can also be considered in the context of the Casimir-Polder force in the system of two atoms. If the atoms are separated by a short distance $d \leqslant r_{0}$ (in a trap, for example), the ZPO are much stronger in the 
interatomic space in comparison with the surrounding space. Then the corresponding Casimir-Polder force should manifest itself by a specific drift of trapped atoms.

In conclusion, the author would like to thank Professor J. H. Eberly and Professor V. I. Rupasov for fruitful discussions.

${ }^{1}$ Long Range Casimir Forces: Theory and Recent Experiments in Atomic Systems, edited by F. S. Levin and D. A. Micha, (Plenum, New York, 1993).

${ }^{2}$ L. Mandel and E. Wolf, Optical Coherence and Quantum Optics (Cambridge University Press, New York, 1995).

${ }^{3}$ M. O. Scully and M. S. Zubairy, Quantum Optics (Cambridge University Press, New York, 1997).

${ }^{4}$ W. H. Louisell, Radiation and Noise in Quantum Electronics (McGrawHill, New York, 1964).
${ }^{5}$ G. Herzberg, Atomic Spectra and Atomic Structure (Dover, New York, 1944); C. E. Moore, Atomic Energy Levels (U.S. National Bureau of Standards, Washington DC, 1971).

${ }^{6} \mathrm{~W}$. Heitler, The Quantum Theory of Radiation (Oxford University Press, New York, 1954); C. Cohen-Tannouji, J. Dupont-Roc, and G. Grinberg, Atom-Photon Interaction (Wiley, New York, 1992).

${ }^{7}$ D. Meschede, H. Walther, and G. Muller, Phys. Rev. Lett. 54, 551 (1985); G. Rempe, H. Walther, and N. Klein, Phys. Rev. Lett. 58, 353 (1987); M. Weidinger, B. T. H. Varcoe, R. Heerlein, and H. Walther, Phys. Rev. Lett. 82, 3795 (1999).

${ }^{8}$ S. Haroche, AIP Conf. Proc. 464, 45 (1999).

${ }^{9}$ L. Mandel, Phys. Rev. 144, 1071 (1966).

${ }^{10}$ D. Kaup and V. I. Rupasov, J. Phys. A 29, 6911 (1996).

${ }^{11}$ A. S. Davydov, Quantum Mechanics (Pergamon, Oxford, 1976).

${ }^{12}$ J. D. Jackson, Classical Electrodynamics (Wiley, New York, 1978).

${ }^{13}$ A. S. Shumovsky and Ö. E. Müstecaplığlu, Phys. Rev. Lett. 80, 1202 (1998). 